\title{
Review
}

\section{Psychological variables associated with quality of life following primary treatment for head and neck cancer: a systematic review of the literature from 2004 to 2015}

\author{
Simon Dunne', Orla Mooney', Laura Coffey ${ }^{2}$, Linda Sharp ${ }^{3}$, Deirdre Desmond ${ }^{2}$, Conrad Timon ${ }^{4}$, Eleanor O'Sullivan ${ }^{5}$ \\ and Pamela Gallagher'* \\ 'Dublin City University, Dublin, Ireland \\ ${ }^{2}$ Maynooth University, Maynooth, County Kildare, Ireland \\ ${ }^{3}$ Newcastle University, Newcastle, UK \\ ${ }^{4}$ St. James's Hospital, Dublin, Ireland \\ ${ }^{5}$ Cork University Dental School and Hospital, Cork, Ireland
}

*Correspondence to:

School of Nursing and Human Sciences, Dublin City University, Glasnevin, Dublin 9, Ireland. E-mail: pamela.gallagher@dcu.ie
Received: 15 October 2015 Revised: 28 January 2016

Accepted: 4 February 2016

\begin{abstract}
Objective: There has been a recent proliferation of research on quality of life (QoL) in head and neck cancer (HNC). The objective of this review was to systematically examine the evidence on psychological factors associated with QoL outcomes for HNC survivors in the post-treatment period published during 2004-2015.

Methods: Five databases were searched for studies investigating psychological factors associated with QoL in HNC survivors. Empirical studies published between January 2004 and June 2015 were included if they measured QoL as an outcome following treatment using a reliable and valid measure, examined its association with at least one psychological factor and included at least 50 HNC survivors.

Results: Twenty-four publications describing 19 studies (9 cross-sectional, 10 prospective) involving 2,263 HNC survivors were included. There was considerable heterogeneity in study design and diversity in measurement and analysis. Distress-related variables (depression, anxiety, distress) were most frequently investigated, and mostly reported negative associations with QoL outcomes. Associations were also observed between other psychological factors (e.g., coping, neuroticism and fear of recurrence) and QoL.

Conclusions: Several psychological factors predict QoL among HNC survivors who have completed treatment. Routine screening and early interventions that target distress could improve HNC survivors' QoL following treatment. Longitudinal and population-based studies incorporating more systematic and standardised measurement approaches are needed to better understand relationships between psychological factors and QoL and to inform the development of intervention and supportive care strategies.

Copyright (C) 2016 John Wiley \& Sons, Ltd.
\end{abstract}

\section{Background}

Head and neck cancer (HNC) refers to a group of related neoplasms of the oral cavity, pharynx, larynx, nasal cavity, middle ear and sinuses [1]. HNC constitutes the seventh most commonly diagnosed cancer and the seventh most common cause of death from cancer worldwide with approximately 686000 new diagnoses and 376000 estimated deaths in 2012 [2]. Treatment for HNC typically involves surgery, radiation therapy, chemotherapy or a combination thereof. Such treatments are often aggressive and can severely impact survivors' quality of life (QoL) [3-5], for example, through visible disfigurement and functional difficulties relating to eating, breathing and speech [6-8].
Quality of life ${ }^{1}$ is a multifaceted construct describing individuals' 'physical health, psychological state, level of independence, social relationships and relationships to salient features in their environment' [9]. QoL is an important outcome in $\mathrm{HNC}[3,4,10,11]$; it assesses the impact of diagnosis and treatment from the perspective of the patient [12] and has a strong positive relationship to survival $[13,14]$. QoL may also be a particularly important outcome for HNC survivorship; research has consistently identified clinical and functional issues that negatively influence QoL following $\mathrm{HNC}$ treatment, including xerostomia, sticky saliva, cancer-related fatigue, visible disfigurement and impairments in speech, taste/smell, swallowing and sexual functioning [8]. Although some of these issues improve over time following HNC 
treatment, many persist 12 months after treatment and can profoundly impact HNC survivors' psychological wellbeing [5]. A recent narrative review highlighted potentially modifiable psychological factors that may be associated with lower QoL among HNC survivors who have finished primary treatment, including heightened distress, body image concerns, fear of recurrence (FoR) and the use of avoidant coping strategies [6]. This research implicates the potential importance of psychological factors in predicting the $\mathrm{QoL}$ of HNC survivors.

A systematic review published in 2005 [15] examining behavioural and psychosocial predictors of QoL in HNC survivors found that psychosocial predictors including depression, personality, social support and satisfaction with consultant information were significantly associated with QoL in this population. The authors underscored the presence of inconclusive findings for certain psychological predictors (e.g. perceived social support) and a dearth of studies on others (e.g. coping), highlighting the need for further high-quality research on psychological predictors of QoL. Since 2005, there has been a proliferation of HNC QoL research. Following these considerations, we undertook a systematic review to examine the current state of the evidence on psychological factors associated with QoL among HNC survivors who had completed their primary treatment. In particular, we aimed to identify the associations between psychological variables (i.e. variables relating to individuals' cognitive and/or emotional processes) measured before, during or after $\mathrm{HNC}$ treatment and QoL outcomes measured following $\mathrm{HNC}$ treatment. Identifying psychological variables associated with post-treatment QoL could aid in the early identification of those at risk for poorer outcomes (e.g. those with particular personality styles) and inform the development of interventions to promote QoL in this population (e.g. by focusing on the training of key psychological skills, which may be modifiable).

\section{Methods}

\section{Search strategy}

In line with preferred reporting items for systematic reviews and meta-analyses (PRISMA) guidelines [16], a systematic search of the literature on QoL in HNC was conducted using five databases: MEDLINE, CINAHL, PsycINFO, EMBASE and Web of Science (all Core Collection: Citation Indexes \& Chemical Indexes). Four of these databases (MEDLINE, CINAHL, PsycINFO and EMBASE) were searched using controlled vocabulary search terms for HNC and QoL. The fifth database, Web of Science, was searched using analogous free text search terms derived from [15] (Supporting Information). All searches were limited to include only literature published in the English language during 01 January 2004 to 08 June 2015. The reference lists of included articles were also manually searched for any additional articles. Figure 1 illustrates the number of papers identified, screened, assessed for eligibility and included in this review.

\section{Selection criteria}

Detailed inclusion and exclusion criteria are provided in Table 1. We defined HNC as neoplasms of the oral cavity, larynx, pharynx, nasal cavity, middle ear and sinuses only. All articles describing studies that exclusively comprised HNC survivors with cancers of the face (e.g. basal cell carcinomas), lip, ears, eyes or thyroid were excluded. This review focussed on survivors in the post-primary treatment phase as this is considered a 'teachable moment' within which interventions to aid psychological well-being may be targeted [3]. All empirical papers investigating the relationship between QoL among HNC patients following primary treatment and at least one psychological variable were deemed eligible; articles that did not clearly report that all participants had received or finished primary treatment for HNC were ineligible. We defined psychological variables as variables comprising individuals' cognitive and/or emotional processes and used this definition to guide the application of our inclusion/exclusion criteria. Following Llewellyn et al. [15], articles were excluded if QoL measurement was limited to functional status, or a measure of disfigurement only, or involved the use of a distress-specific instrument such as the Hospital Anxiety and Depression Scale (HADS).

\section{Data extraction}

Titles and abstracts of identified articles were independently screened for eligibility by two authors (S.D. and O.M.). Full texts of potentially eligible articles were obtained and assessed for eligibility by each reviewer according to the aforementioned criteria. Any discrepancies were resolved through discussion and/or consultation with the third author (P.G.). The following data were extracted from eligible articles: (1) study location; (2) aim(s); (3) design; (4) recruitment setting; (5) survivor characteristics (age, gender, site of tumour and treatment received); (6) QoL tool used; (7) psychological predictors assessed (and tools used); (8) assessment time-points; (9) whether or not psychological factors or their sub-components (e.g. neuroticism) were associated with QoL in cross-sectional analyses (and, if so, the specific results in the overall study population and subgroups considered); and (10) whether or not psychological factors or their sub-components were associated with QoL in prospective analyses (and, if so, the specific results). Where available, results derived from multiple regression models were extracted. Otherwise, unadjusted results were abstracted.

\section{Quality assessment}

Eligible articles were critically appraised by two authors (S.D. and L.C.) to evaluate their risk of bias using a 


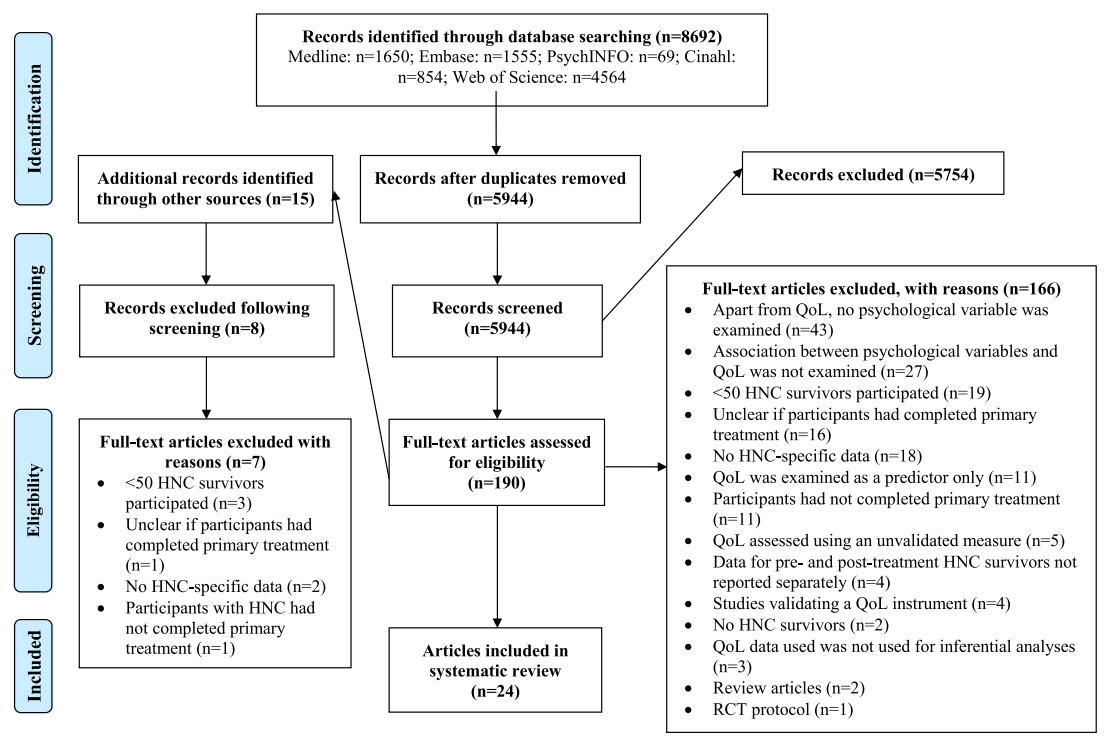

Figure I. Papers identified, screened, assessed for eligibility and included for review

Table I. Article inclusion/exclusion criteria

\begin{tabular}{|c|c|}
\hline Inclusion criteria & Exclusion criteria \\
\hline $\begin{array}{l}\text { 1. Articles describing empirical studies with HNC survivors with a } \mathrm{Q} \mathrm{OL} \text { measurement } \\
\text { instrument that was recognised as a reliable and valid measure and provided a measure } \\
\text { of global QoL. }\end{array}$ & $\begin{array}{l}\text { I. Variables associated with QoL were clinical or related to } \\
\text { symptoms (e.g. pain and xerostomia). }\end{array}$ \\
\hline 3. QoL was measured following primary treatment. & $\begin{array}{l}\text { 3. Studies exclusively comprising participants receiving } \\
\text { palliative care. }\end{array}$ \\
\hline 5. Studies with QoL outcome data for $N \geq 50 \mathrm{HNC}$ patients. & $\begin{array}{l}\text { 5. Non-English language studies. } \\
\text { 6. Primary aim of the study was QoL instrument validation. }\end{array}$ \\
\hline
\end{tabular}

HNC, head and neck cancer; QoL, quality of life.

12-item checklist, which was based on a checklist from previous research $[17,18]$ supplemented by standard critical appraisal questions [19]. Articles were given a score of 2 for items marked as 'Yes', 1 for items marked as 'Partially' and 0 for items marked as 'No' on the checklist. Articles were considered to have good quality if they scored 17 or more out of a maximum possible score of 24 , adequate quality if they scored $9-16$ and poor quality if they scored $0-8$. Where disagreement between the appraising authors arose, consensus was achieved through discussion. A third author (P.G.) was consulted if disagreement persisted.

\section{Statistical analysis}

Due to heterogeneity in the analyses between the studies described in the included articles, meta-analysis was not undertaken.

\section{Results}

Following database searching and initial screening of titles and abstracts, the full texts of 190 articles were assessed for eligibility. Of these, 24 articles reporting 19 studies fulfilled the inclusion criteria. Characteristics of, and summary results from, these studies [20-43] are provided in Table 2. A full description of results of these studies is provided in Table $\mathrm{S} 1$.

\section{Study characteristics}

The 19 studies (described in 24 articles) included 2347 patients, of whom 673 (29\%) were women. Only 16 studies reported participants' age $(M=61$ years, range $=$ 23-94). Sample sizes ranged from 51 to 376 and included HNC survivors with cancers affecting the oral cavity $(n=$ 16), pharynx $(n=15)$, larynx $(n=12)$, tongue $(n=6)$, 


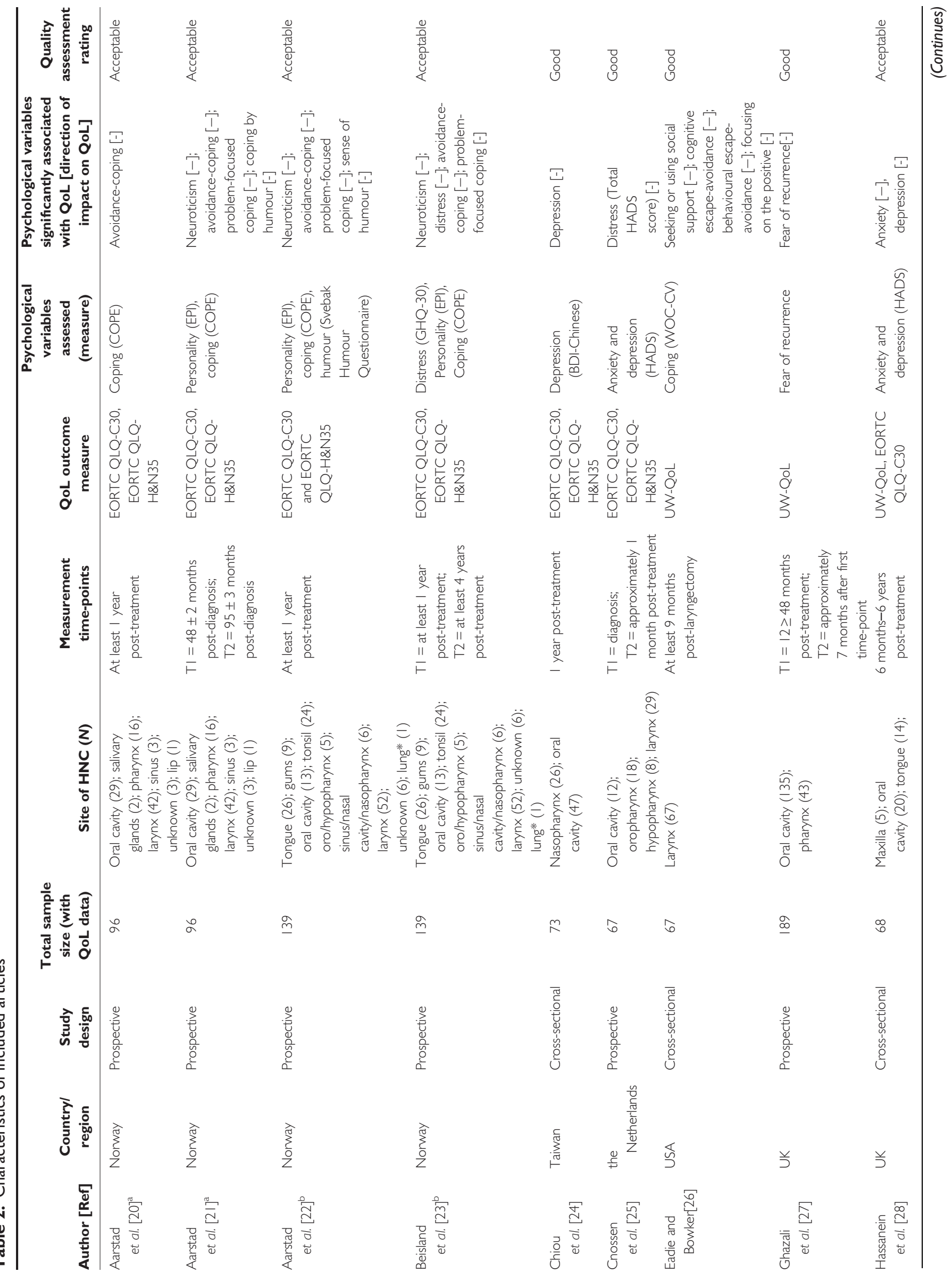




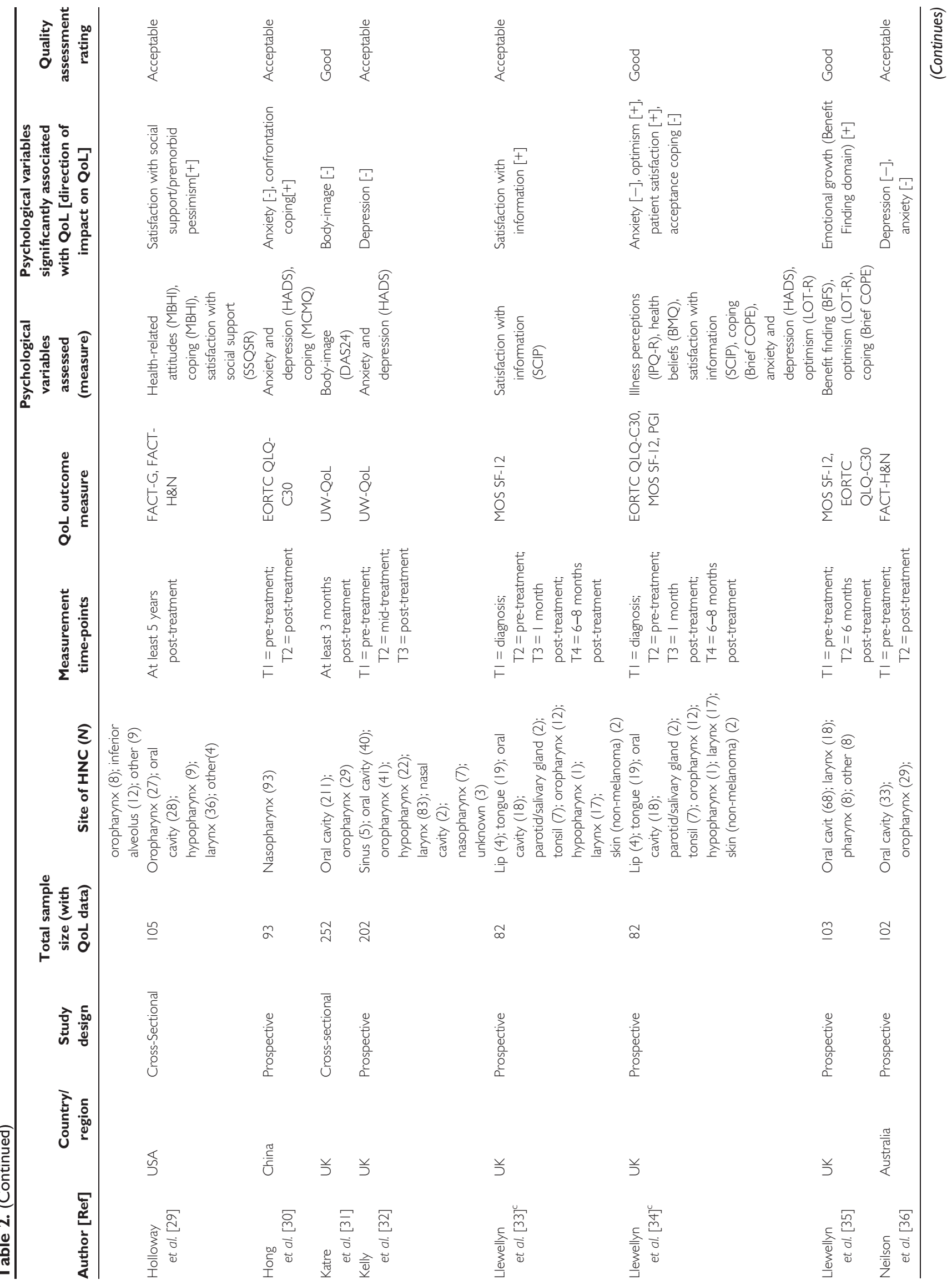




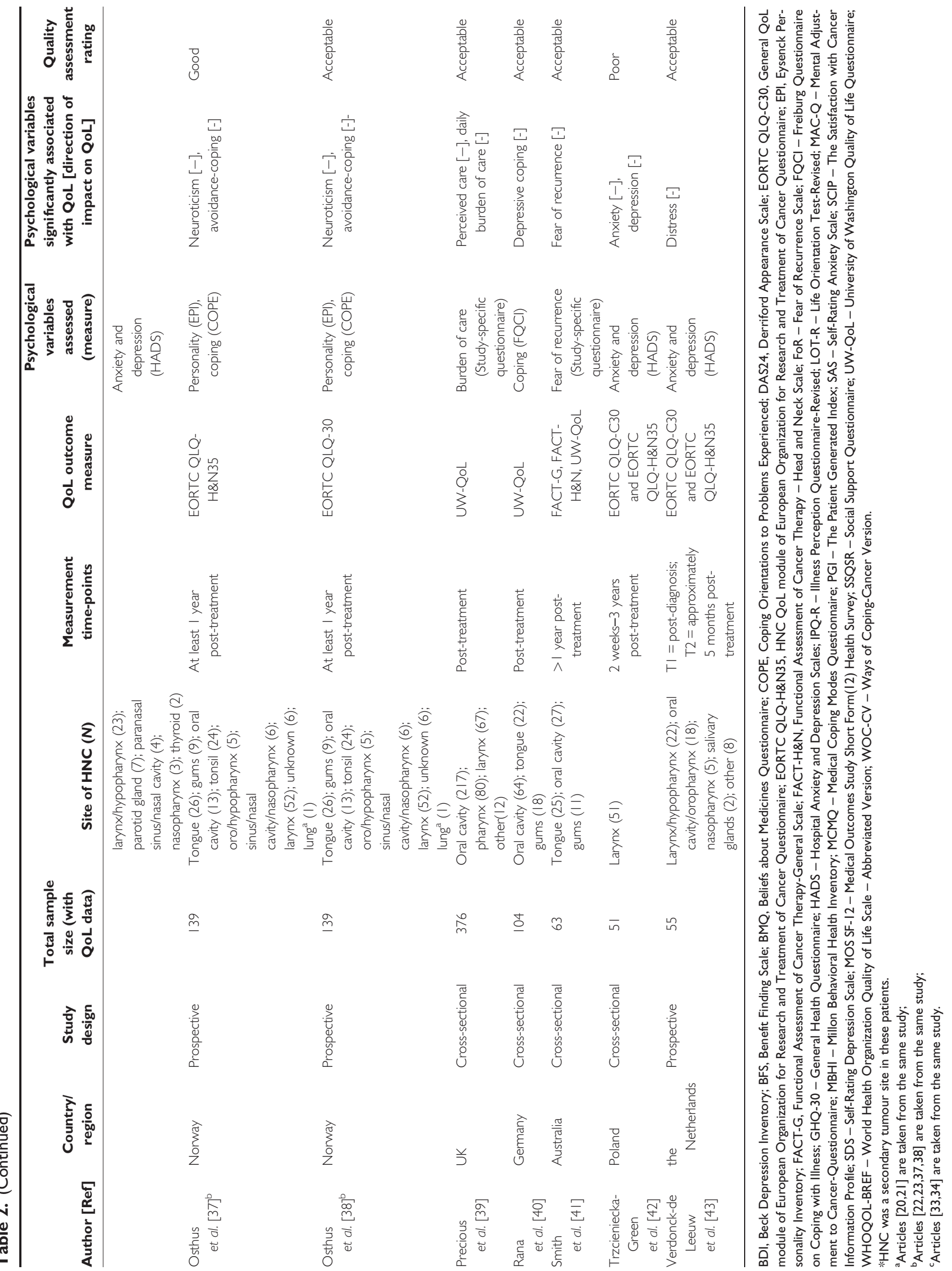


sinuses $(n=4)$, gums $(n=3)$, salivary glands $(n=3)$, nasal cavity $(n=3)$, inferior alveolus $(n=1)$ and/or unknown or unspecified primary $\mathrm{HNC}(n=13)$. At the time of final QoL assessment, HNC survivors had completed their primary cancer treatment from 1 month to 7 years previously. The eligible articles included cross-sectional $(n=9)$ and prospective $(n=10)$ designs. ${ }^{2}$ Studies were conducted in eight countries/regions: UK $(n=7)$, Norway $(n=2)$, USA $(n=2)$, Australia $(n=2)$, the Netherlands $(n=2)$, Germany $(n=1)$, China $(n=1)$, Poland $(n=1)$ and Taiwan $(n=1)$. Participants had received surgery, radiation, chemotherapy or a combination thereof $(n=9)$, surgery and/or radiation $(n=6)$, chemotherapy and/or radiation $(n=1)$, surgery only $(n=1)$, radiation only $(n=1)$ or treatment was not specified $(n=1)$. Participants were recruited from either single clinics $(n=$ $14)$, multiple clinics $(n=4)$ or support groups and professional contacts $(n=1)$.

There was considerable heterogeneity in the questionnaires used to measure QoL: the general QoL module of the European Organization for Research and Treatment of Cancer Questionnaire (EORTC QLQ-C30; $n=13$ ), EORTC HNC QoL (EORTC QLQ-H\&N35; $n=9$ ), University of Washington Quality of Life Questionnaire (UW-QoL; $n=7$ ), Functional Assessment of Cancer Therapy - Head and Neck Scale (FACT-HN; $n=3)$, Medical Outcomes Study Short Form (12) Health Survey (MOS SF-12; $n=3$ ), FACT General Scale (FACT-G; $n=2$ ), World Health Organization Quality of Life Scale Abbreviated Version (WHOQoL-Bref; $n=1$ ) and/or Patient Generated Index (PGI; $n=1$ ). There was also considerable diversity in the psychological variables examined and the assessment instruments used to measure them. Distress-related variables (i.e. depression, anxiety or combined distress scores) were the most commonly assessed psychological factors $(n=10)$ and were measured using the HADS, Beck Depression Inventory (BDI), General Health Questionnaire (GHQ), Self-Rating Depression Scale (SDS) or Self-Rating Anxiety Scale (SAS). Coping was examined in six studies with the Coping Orientations to Problems Experienced (COPE), Brief COPE, Ways of Coping - Cancer Version (WOC-CV), Medical Coping Modes Questionnaire (MCMQ) or Freiburg Questionnaire on Coping with Illness (FCQI). Personality was assessed in five studies with the Eysenck Personality Inventory (EPI), Life Orientation Test - Revised (LOT-R) or Millon Behavioral Health Inventory (MBHI). HNC survivors' perceptions of the care they received was examined in four studies, using the Satisfaction with Information Profile (SCIP; $n=2$ ), the Social Support Questionnaire (SSQ; $n=1$ ) and three non-validated questions relating to perceived burden of care in the remaining study [39]. Fear of recurrence was assessed in two studies with two different unvalidated measures [24,38]. Two additional psychological variables were assessed in a single study each: body image using the Derriford Appearance Scale
(DAS24) and benefit-finding (i.e. seeking benefit from adverse experiences) using the Benefit Finding Scale (BFS).

All included studies demonstrated a significant relationship between at least one psychological factor and posttreatment QoL outcomes. Of the 15 articles describing 10 studies with prospective designs, only six articles describing five studies [21,23,27,30,33,34] reported longitudinal analyses in relation to psychological predictors of QoL (i.e. where the psychological factor was measured before the QoL outcome), while the other nine articles describing seven studies [20,22,25,32,35-38,43] reported only cross-sectional associations between psychological factors and QoL in the post-treatment period. The remaining nine articles describing nine studies $[24,26,28,29,37,39-42]$ reported on cross-sectional associations between at least one psychological factor and post-treatment QoL outcomes.

\section{Cross-sectional analyses}

\section{Distress-related variables}

The cross-sectional relationship between depression and QoL was examined in five studies [24,28,32,36,42]. In each study, higher depression scores among HNC survivors were significantly associated with poorer QoL outcomes, including lower physical, mental and social functioning and higher generic and HNC-specific symptom scores in four studies [24,28,36,42]; lower emotional well-being in three studies [24,28,32]; and lower global or overall QoL in two studies $[24,28]$. Four studies also examined the relationship between anxiety and QoL among HNC survivors cross sectionally $[28,30,36,42]$. Higher levels of anxiety were associated with higher generic and $\mathrm{HNC}$-specific symptom scores in three studies [28,36,42], lower social functioning in two studies $[28,42]$, lower overall QoL in two studies [28,30] and lower physical functioning, mental functioning and emotional wellbeing scores in one study [28]. A significant interaction between anxiety and gender in relation to QoL among nasopharyngeal carcinoma survivors was observed in one study [30]; anxious females (who scored $>11$ on the anxiety subscale of the HADS) exhibited significantly lower QoL compared with non-anxious women, while anxious men did not display significantly lower QoL than non-anxious men. Finally, the relationship between distress (combined anxiety and depression scores) and QoL was examined in three studies $[23,25,43]$. Higher distress was significantly associated with higher HNC-specific symptom scores in all three studies $[23,25,43]$ as well as lower physical functioning and emotional well-being scores and higher generic symptom scores in two studies $[23,43]$.

\section{Coping}

Five studies examined associations between coping and QoL through cross-sectional analyses [20-23,26,30,37,38,40]. In two studies using the COPE, avoidance coping was 
associated with poorer QoL outcomes, including lower functioning and global QoL [20-23,37,38] and higher generic and HNC-specific symptom scores [20-23], while problem-focused coping was associated with poorer functional QoL, general cancer symptom QoL and HNC-specific symptom QoL [20-23]. In one of these studies, 'drinking to cope' was negatively associated with global and functional QoL [21]; in the other, coping through humour was negatively associated with functional QoL outcomes and positively associated with general and HNC-specific symptoms [22]. In a study using the MCMQ [30], lower confrontation coping was associated with poorer total QoL scores. Another study found that all five patterns of coping from the WOCCV (seeking or using social support, distancing, cognitive escape-avoidance, behavioural escape-avoidance and focusing on the positive) were associated with poorer QoL outcomes among laryngectomy survivors [26]. Finally, one study [40] found that 'depressive coping' measured with the FCQI had a significant negative association with overall QoL.

\section{Personality}

Two studies assessed personality using the EPI [20$23,37,38]$. In both studies, cross-sectional analyses indicated that high neuroticism scores were associated with poorer QoL outcomes in all domains of both EORTC QoL scales. Additionally, in both studies, greater neuroticism was a significant independent predictor of lower functional QoL and higher HNC-specific symptoms [21,23].

\section{Perceptions of care received}

Four studies examined the relationship between QoL and variables, which measured HNC survivors' perceptions of the care they received, specifically, survivors' satisfaction with their care [33,34], perceived burden on carers [39] and satisfaction with the social support they received [29]. The findings of two articles derived from the same study $[33,34]$ indicated that higher levels of patient satisfaction with information on illness and treatment measured before and after treatment predicted better mental functioning QoL. In another study [39], a range of variables measuring survivors' perceptions of the burden of care they placed on their caregiver were related to poorer functional QoL outcomes; that is, patients with poorer functioning felt they were a greater burden on their carers. Finally, in one study [29], a combination of greater satisfaction with social support (as measured by the SSQ) and lower premorbid pessimism (as measured by the MBHI) predicted higher social/family well-being among this cohort.

\section{Fear of recurrence}

Fear of recurrence and QoL were assessed in cross-sectional analyses in two studies [27,41]. In one study, greater FoR (assessed using a single item) was significantly associated with lower overall QoL [41]. In the other, greater FoR (assessed using a seven-item questionnaire) was associated with lower social and emotional functioning [27].

\section{Other psychological factors}

In one study [31], greater body image concerns were associated with poorer QoL in all domains of the UW-QoL. One further study [35] found that the emotional and spiritual growth item in the benefit-finding scale (i.e. deriving emotional or spiritual growth from HNC) was associated with higher levels of mental health-related QoL.

\section{Longitudinal analyses}

Five studies [21,23,27,30,33,34] provided longitudinal analyses of psychological factors and QoL. Two of these studies examined the relationship between anxiety (measured by the HADS) and subsequent QoL. In one study [30], HNC survivors with high levels of anxiety before radiotherapy had the greatest decline in QoL following treatment. The other study [34] found that anxiety, optimism (measured by the LOT-R) and patient satisfaction at diagnosis predicted mental functioning QoL scores 68 months following treatment. The longitudinal association between distress and QoL was also examined in one study [23]; distress levels (measured by the GHQ) at least 1 year post-treatment were associated with poor QoL outcomes among HNC survivors 3 years later. Two studies $[21,23]$ investigated the relationship between personality and coping (measured by the EPI and COPE, respectively) after treatment and subsequent QoL outcomes. Both studies reported that higher levels of neuroticism, problem-focused coping and avoidance-focused coping immediately after treatment predicted lower functional and global QoL and higher generic and HNCspecific symptoms 3 or more years later. One of these studies [21] also found that 'drinking to cope' and 'coping by humour' measured 4 years post-treatment were associated with poor QoL outcomes up to 4 years later. One additional study, which examined the longitudinal relationship between coping and QoL, found that acceptance coping at diagnosis (measured by the Brief COPE) predicted global QoL 6-8 months following treatment when combined with tumour stage and pre-treatment QoL [34]. Two studies [33,34] found that higher levels of patient satisfaction at diagnosis (measured by SCIP) predicted better mental functioning QoL 6-8 months following treatment. Finally, one study [27] found that an increase in FoR from an initial post-treatment visit to approximately 7 months later was associated with worsening anxiety-related QoL scores at least 7 months later.

\section{Quality assessment}

The quality assessment is summarised in Table 1 (with further details in Table S2). Eight articles were scored as 
'good quality', 15 were rated as 'adequate quality' and one was rated as 'poor quality'. Areas in which articles scored poorly included failure to justify sample size, not having a control group, not justifying statistical practices (e.g. dichotomizing scores on psychological variables into binary predictors without providing a rationale) and poor descriptions of the population, design, recruitment strategy and characteristics of non-responders/nonparticipants.

\section{Conclusions}

The current review builds on the findings of a prior systematic review [15] to demonstrate that several key psychological factors significantly predict QoL among HNC survivors. As observed previously, depression and neuroticism were important predictors of negative QoL outcomes in $\mathrm{HNC}$ research published in the last 10 years. The current review extends the findings of this previous review by providing evidence that anxiety, distress, FoR and coping style are also strongly associated with poor QoL outcomes among HNC survivors following treatment. In line with this prior review [15], two measures of perceptions of care received, patient satisfaction $[41,42]$ and satisfaction with social support [29], were also associated with positive QoL outcomes. However, the limited evidence available suggests that further investigation of these associations is warranted. Some limited data from individual studies also suggest that other psychological variables such as body image [31], benefit-finding [35] and perceived burden of care [39] may have significant associations with QoL. These findings, together with the wider body of literature on these variables [e.g. 4446], suggest promising avenues for further research and the potential for future intervention development among HNC survivors. In particular, it is likely that body image may be an important predictor of QoL outcomes among this cohort given the potential for visible disfigurement following $\mathrm{HNC}$ treatment. Nonetheless, the quality assessment indicated a number of shortcomings in many studies, including limited description of the population, design and recruitment strategies, limited justification of statistical practices and potential bias in the reporting of significant findings; suggesting that, a decade on from the review of Llewellyn et al. [15], there remains limited high-quality research investigating the psychological factors that predict QoL among HNC survivors. Furthermore, the findings from the quality assessment mean that the study results should be interpreted with caution and care taken when considering the full implications of the findings described herein.

In this review, distress-related variables were consistently associated with poorer QoL outcomes among post-treatment HNC survivors in cross-sectional and longitudinal analyses. Prior to 2005, research investigating the impact of distress-related variables on QoL in $\mathrm{HNC}$ typically focused on depression [15], and variables such as anxiety and emotional distress had rarely been examined. Consistent with the findings of this research, in the current review, depression was associated with poorer QoL outcomes in cross-sectional analyses $[24,28,32,36,42]$. A novel finding of this review is that higher anxiety levels were similarly associated with a range of poorer QoL outcomes across five studies $[28,30,34,36,42]$, and distress (combined anxiety and depression scores) was associated with impaired QoL among HNC survivors in three studies [22,25,43]. These findings are consistent with the broader cancer survivorship literature, where both depression and anxiety have been associated with lower QoL among diverse groups of cancer survivors including those with breast, colorectal and lung cancer [47-49]. However, it should be noted that clinical assessments of depression, anxiety and/or distress were not undertaken in these studies. In line with recommendations from prior systematic reviews and clinical guidelines for HNC care pathways [50-53], routine clinical screening for both depression and anxiety (or distress) by practitioners could enable the identification of individuals at risk for poorer QoL outcomes following HNC treatment and who might benefit from intervention and/or support. In particular, while depression and anxiety are known to occur at all stages of the disease trajectory among HNC survivors [6], it may be important to identify individuals who exhibit clinical symptoms of distress post-treatment and may benefit from formal psychological support [50].

A further novel finding of the current review is the presence of strong negative associations between FoR and post-treatment QoL outcomes across two studies $[27,43]$. FoR represents a particularly important area for study among this survivor population given that recurrence risk is high in HNC [54-56]. While the current findings need replication in larger populations, employing more standardised and psychometrically robust measures of this construct (such as the Fear of Progression Questionnaire or the shorter Assessment of Survivor Concerns Scale [57]) would also be useful. It has been estimated that between half and three-quarters of this population have concerns about recurrence [6]. Interventions that target the management of FoR concerns (e.g. through active emotion-focused and behavioural strategies to manage uncertainty fears) may increase QoL among HNC survivors where recurrence fears have become intrusive [58,59]. Indeed, such interventions have been successful among other groups of cancer survivors [e.g. 59].

This review also reveals a striking negative relationship between the use of specific coping strategies and QoL outcomes among HNC survivors. Indeed, a range of both active (coping with humour, seeking or using social support, focusing on the positive and acceptance coping) 
and avoidant coping strategies (coping by suppression of competing activities, coping by behavioural avoidance, drinking to cope, cognitive escape-avoidance, behavioural escape-avoidance, low confrontation coping and depressive coping) were associated with poorer QoL outcomes among HNC survivors in both cross-sectional and longitudinal analyses [20-23,26,30,34,37,38,40]. It is unclear exactly why both active and avoidant coping strategies are associated with poorer QoL outcomes among HNC survivors, but interestingly, similar associations have been reported among prostate cancer survivors (e.g. [60,61]). Aarstad et al. [21] propose that such findings may indicate that HNC survivors who perceive they have poorer QoL employ more attempts to cope with these challenges, thereby more vigorously applying a variety of coping strategies. While this cannot explain longitudinal associations between active and avoidant coping strategies and subsequent QoL, it should be noted that the two studies in the current review, which examined longitudinal associations between active and avoidant coping strategies and QoL [21,23], did not measure coping strategies at more than one time point, and it is possible that coping strategies employed by HNC survivors may change over time. Because emerging research has indicated that the QoL of HNC survivors may stabilise or improve beyond baseline levels approximately 5 years after $\mathrm{HNC}$ treatment $[62,63]$ or may deteriorate over time due to late effects $[64,65]$, it would be useful to establish whether individual active and avoidant coping strategies interact with QoL outcomes over time in order to identify time points across the posttreatment trajectory when interventions to improve HNC survivors' coping skills would be most appropriate.

The findings from the studies included in this review give a somewhat more limited picture of the relationship between personality variables and QoL. Neuroticism was associated with lower QoL outcomes in both crosssectional and longitudinal analyses across two studies from a Norwegian research group [21-23,37,38]. Together with the findings of the review of Llewellyn et al. [15], these results point towards a consistent association between neuroticism and poor QoL outcomes among HNC survivors. In terms of potential explanations for this relationship, neuroticism is typically associated with more negative health perceptions generally (e.g. [66-68]). In one study [34], optimism measured at diagnosis was associated with improved mental functioning QoL following treatment. However, other personality variables such as agreeableness, conscientiousness and impulsivity have not been investigated, suggesting that further research is needed to identify whether additional personality factors such as these may affect the QoL of HNC survivors following treatment.

A major finding of the current review is the wide heterogeneity in measurement, sampling and design between studies; indeed, the heterogeneity was so great as to preclude formal statistical combination of the studies. In general, there was great diversity in QoL measures used, and these different QoL measures incorporate specific sub-domains of QoL, which cannot be easily compared as they may be tapping into different aspects of QoL. There is currently no recognised gold standard measure for QoL in HNC [10]; future research would benefit from the development of more standardised approaches to $\mathrm{QoL}$ measurement among this population. There was also diversity in the sample size and composition of included studies. Notably, sample sizes were relatively small, with the largest study including less than 400 participants. Furthermore, recruitment strategies were not documented in many studies and most recruited from single (or a small number) of clinics, raising the possibility that results may not be generalizable to the wider population of HNC survivors. Larger population-based studies investigating the psychological factors that predict QoL outcomes among HNC survivors are needed to establish the veracity of the findings described herein, to ensure adequate power and enable informative analyses of HNC subgroups or interactions between psychological and other predictors of QoL. Finally, it should be noted that several studies included in the current review reported only modest associations between psychological variables and QoL. Greater efforts should be made in future studies to provide further information on design and recruitment strategies, justify statistical practices, discuss non-significant findings in detail and clarify the strength of associations between psychological variables and QoL in order to enhance their replicability and demonstrate that they are free from bias.

While this review has identified important directions for future HNC survivorship research and intervention development, it has a number of limitations. Firstly, the review is limited to studies published in the English language. There may be additional relevant research that has been published in other languages. Additionally, this review is restricted to examining psychological predictors of QoL in the post-treatment period alone, and the findings may not apply to HNC patients undergoing or awaiting treatment. However, a wealth of evidence suggests that the post-primary treatment phase is a unique period of intense vulnerability for HNC survivors, characterised by lower QoL [5,6,11], and thus merits individual attention.

In conclusion, this review shows that there are strong negative associations between distress-related variables and the QoL of HNC survivors following their treatment. Interventions to reduce distress may help to improve $\mathrm{HNC}$ survivors' QoL, and routine screening for distress may identify those at risk for poor QoL in this period. However, there is a need for further longitudinal and population-based studies, which take more systematic and standardised measurement approaches to better understand the relationship between QoL and other 
psychological variables among post-treatment HNC survivors. Such studies could inform the development of interventions and supportive care strategies to improve QoL in this population.

\section{Notes}

1. In clinical practice, QoL typically refers to healthrelated QoL, which comprises aspects of QoL related to health or medical concerns, and measures physical, psychological and social domains [15]. For this review, we have opted for the more general term 'quality of life', which may refer to both QoL and health-related QoL, as these terms are often used interchangeably in the literature.

2. Studies were classified as prospective if at least one psychological factor was measured at a time point that preceded the QoL measurement.

\section{References}

1. National Cancer Registry Ireland. Cancer Trends No 10. Cancers of the head and neck [Internet]. 2011. Available from: http://www. ncri.ie/sites/ncri/files/pubs/CancerTrendsNo. 10-CancersoftheHeadandNeck.pdf [accessed 26 August 2015]

2. Ferlay J, Soerjomataram I, Dikshit R, et al. Cancer incidence and mortality worldwide: sources, methods and major patterns in GLOBOCAN 2012. Int $J$ Cancer 2015;136: E359-E386.

3. Murphy BA, Ridner S, Wells N, Dietrich M. Quality of life research in head and neck cancer: a review of the current state of the science. Crit Rev Oncol Hematol 2007;62:251-267.

4. Sayed SI, Elmiyeh B, Rhys-Evans P, et al. Quality of life and outcomes research in head and neck cancer: a review of the state of the discipline and likely future directions. Cancer Treat Rev 2009;35:397-402.

5. So WKW, Chan RJ, Chan DNS, et al. Quality of life among head and neck cancer survivors at one year after treatment - a systematic review. Eur J Cancer 2012;48:2391-2408.

6. Howren MB, Christensen AJ, Karnell LH, Funk GF. Psychological factors associated with head and neck cancer treatment and survivorship: evidence and opportunities for behavioural medicine. J Consult Clin Psychol 2013;81:299-317.

7. Djan R, Pennington A. A systematic review of questionnaires to measure the impact of appearance on quality of life for head and neck cancer patients. J Plast Reconstr Aesthet Surg 2013;66:647-659.

8. Van der Molen L, van Rossum MA, Burkhead LM, Smeele LE, Hilgers FJM. Functional outcomes and rehabilitation strategies in patients treated with chemoradiotherapy for advanced head and neck cancer: a systematic review. Eur Arch Otolaryngol 2009;266:889-900.

9. World Health Organization Working Groups. Programme on Mental Health: WHOQOL Measuring Quality of Life, World Health Organization: Geneva, 1997;1.

10. Babin E, Sigston E, Hitier M, Dehesdin D, Marie JP, Choussy O. Quality of life in head and neck cancer patients: predictive factors, functional and psychosocial outcome. Eur Arch Otolaryngol 2008;265:265-270.
11. Singer S, Langendijk J, Yarom N. Assessing and improving quality of life in patients with head and neck cancer. Am Soc Clin Oncol Educational Book 2013. DOI:10.1200/EdBook_ AM.2013.33.e230.

12. Humphris GM. The missing member of the head and neck multidisciplinary team: the psychologist. Why we need them. Curr Opin Otolaryngol Head Neck Surg 2008; 16:108-112.

13. Van Nieuwenhuizen AJ, Buffart LM, Brug J, Leemans CR, Verdonck-de Leeuw IM. The association between health related quality of life and survival in patients with head and neck cancer: a systematic review. Oral Oncol 2015;51:1-11.

14. Karvonen-Gutierrez CA, Ronis DL, Fowler KE, Terrell JE, Gruber SB, Duffy SA. Quality of life scores predict survival among patients with head and neck cancer. J Clin Oncol 2008;26:2754-2760.

15. Llewellyn CD, McGurk M, Weinman J. Are psycho-social and behavioural factors related to health-related quality of life in patients with head and neck cancer? A systematic review. Oral Oncol 2005;41:440-454.

16. Moher D, Liberati A, Tetzlaff J, Altman DG, The PRISMA Group. Preferred reporting items for systematic reviews and metaanalyses: the PRISMA statement. J Clin Epidemiol 2009;62:1006-1012.

17. Jefferies P, Gallagher P, Dunne S. The Paralympic athlete: a systematic review of the psychosocial literature. Prosthet Orthot Int 2012;3:278-289.

18. O'Connor M, Gallagher P, Waller J, Martin CM, O'Leary JJ, Sharp L, and on behalf of the Irish Cervical Screening Research Consortium (CERVIVA). Adverse psychological outcomes following colposcopy and related procedures: a systematic review. BJOG 2015 [epub ahead of print]. DOI:10.1111/14710528.13462

19. Crombie IK. The Pocket Guide to Critical Appraisal: A Handbook for Health Care Professionals, Chapter 6, BMJ Publishing Group: London, 1996;23-29.

20. Aarstad AKH, Aarstad HJ, Bru E, Olofsson J. Psychological coping style versus disease extent, tumour treatment and quality of life insuccessfully treated head and neck squamous cell carcinoma patients. Clin Otolaryngol 2005;30:530-538.
21. Aarstad AKH, Aarstad HJ, Olofsson J. Personality and choice of coping predict quality of life in head and neck cancer patients during follow-up. Acta Oncol 2008;47:879-890.

22. Aarstad AKH, Beisland E, Osthus AA, Aarstad HJ. Distress, quality of life, neuroticism and psychological coping are related in head and neck cancer patients during followup. Acta Oncol 2011;50:390-398.

23. Beisland E, Aarstad AKH, Osthus AA, Aarstad HJ. Stability of distress and healthrelated quality of life as well as relation to neuroticism, coping and TNM stage in head and neck cancer patients during follow-up. Acta Otolaryngol 2013;133:209-217.

24. Chiou WY, Lee MS, Ho HC, et al. Prognosticators and the relationship of depression and quality of life in head and neck cancer. Indian J Cancer 2013;50:14-20.

25. Cnossen IC, de Bree R, Rinkel RNPM, et al. Computerized monitoring of patient-reported speech and swallowing problems in head and neck cancer patients in clinical practice. Support Care Cancer 2012;20:2925-2931.

26. Eadie TL, Bowker BC. Coping and quality of life after total laryngectomy. Otolaryngol Head Neck Surg 2012;146:959-965.

27. Ghazali N, Cadwallader E, Lowe D, Humphris G, Ozakinci G, Rogers SN. Fear of recurrence among head and neck cancer survivors: longitudinal trends. Psychooncology 2013;22:807-813.

28. Hassanein KAM, Musgrove BT, Bradbury E. Psychological outcome of patients following treatment of oral cancer and its relation with functional status and coping mechanisms. $J$ Cranio-Maxillofac Surg 2005;33:404-409.

29. Holloway RL, Hellewell JL, Marbella AM, Layde PM, Myers KB, Campbell BH. Psychosocial effects in long-term head and neck cancer survivors. Head Neck 2005;27:281-288.

30. Hong J, Tian J, Zhang W, et al. Patient characteristics as indicators for poor quality of life after radiotherapy in advanced nasopharyngeal cancer. Head Neck Oncol 2013;5:17.

31. Katre C, Johnson IA, Humphris GM, Lowe D, Rogers SN. Assessment of problems with appearance, following surgery for oral and oro-pharyngeal cancer using the University of Washington appearance domain and the Derriford Appearance Scale. Oral Oncol 2008;44:927-934.

32. Kelly C, Paleri V, Downs C, Shah R. Deterioration in quality of life and depressive 
symptoms during radiation therapy for head and neck cancer. Otolaryngol Head Neck Surg 2007;136:108-111.

33. Llewellyn CD, McGurk M, Weinman J. How satisfied are head and neck cancer (HNC) patients with the information they receive pretreatment? Results from the satisfaction with information profile (SCIP). Oral Oncol 2006;42:726-734.

34. Llewellyn CD, McGurk M, Weinman J. Illness and treatment beliefs in head and neck cancer: is Leventhal's common sense model a useful framework for determining changes in outcomes over time? $J$ Psychosom Res 2007; 63:17-26.

35. Llewellyn CD, Horney DJ, McGurk M, et al. Assessing psychological predictors of benefit finding in patients with head and neck cancer. Psychooncology 2013;22:97-105.

36. Neilson KA, Pollard AC, Boonzanier AM, et al. Psychological distress (depression and anxiety) in people with head and neck cancers. Med J Aust 2010;193:S48-S51.

37. Osthus AA, Aarstad AKH, Olofsson J, Aarstad HJ. Head and neck specific health related quality of life scores predict subsequent survival in successfully treated head and neck cancer patients: a prospective cohort study. Oral Oncol 2011;47:974-979.

38. Osthus AA, Aarstad AKH, Olofsson J, Aarstad HJ. Health-related quality of life scores in long-term head and neck cancer survivors predict subsequent survival: a prospective cohort study. Clin Otolaryngol 2011;36:361-368.

39. Precious E, Haran S, Lowe D, Rogers SN Head and neck cancer patients' perspective of carer burden. Br J Oral Maxillofac Surg 2012;50:202-207.

40. Rana M, Kanatas A, Herzberg PY, et al. Prospective study of the influence of psychological and medical factors on quality of life and severity of symptoms among patients with oral squamous cell carcinoma. $\mathrm{Br} J$ Oral Maxillofac Surg 2015;53:364-370.

41. Smith GI, Yeo D, Clark J, et al. Measures of health-related quality of life and functional status in survivors of oral cavity cancer who have had defects reconstructed with radial forearm free flaps. Br J Oral Maxillofac Surg 2006;44:187-192.

42. Trzcieniecka-Green A, Bargiel-Matusiewicz K, Borczyk J. Quality of life of patients after laryngectomy. $J$ Physiol Pharmacol 2007;58(Suppl 5(Pt 2)):699-704.

43. Verdonck-de Leeuw IM, de Bree R, Keizer AL, et al. Computerized prospective screening for high levels of emotional distress in head and neck cancer patients and referral rate to psychosocial care. Oral Oncol 2009;45:e129-e133.
44. Rhoten BA, Murphy B, Ridner SH. Body image in patients with head and neck cancer: a review of the literature. Oral Oncol 2013;49:753-760.

45. Rajandram RK, Jenewein J, McGrath C, Zwahlen RA. Coping processes relevant to posttraumatic growth: an evidence-based review. Support Care Cancer 2011;19:583-589.

46. Harrington S, McGurk M, Llewellyn CD. Positive consequences of head and neck cancer: key correlates of finding benefit. $J$ Psychosoc Oncol 2008;26:43-62.

47. Hutter N, Vogel B, Alexander T, Baumeister $\mathrm{H}$, Helmes A, Bengel J. Are depression and anxiety determinants or indicators of quality of life in breast cancer patients? Psychol Health Med 2013;18:412-419.

48. Graça Pereira M, Figueiredo AP, Fincham FD. Anxiety, depression, traumatic stress and quality of life in colorectal cancer after different treatments: a study with Portuguese patients and their partners. Eur J Oncol Nurs 2012;16:227-232.

49. Arrieta O, Angulo LP, Núñez-Valencia C, et al. Association of depression and anxiety on quality of life, treatment adherence, and prognosis in patients with advanced nonsmall cell lung cancer. Ann Surg Oncol 2013;20:1941-1948.

50. Luckett T, Britton B, Clover K, Rankin NM. Evidence for interventions to improve psychological outcomes in people with head and neck cancer: a systematic review of the literature. Support Care Cancer 2011;19:871-881.

51. National Comprehensive Cancer Network $(\mathrm{NCCN})$. NCCN practice guidelines for the management of psychosocial distress. Oncology 1999;13:113-147.

52. Semple C, Parahoo K, Norman A, McCaughan E, Humphris G, Mills M. Psychosocial interventions for patients with head and neck cancer. Cochrane Database Syst Rev 2013;7CD009441:. DOI:10.1002/ 14651858.CD009441.pub2.

53. Krebber AH, Jansen F, Cuijpers P, Leemans CR, Verdonck-de Leeuw IM. Screening for psychological distress in follow-up care to identify head and neck cancer patients with untreated distress. Support Care Cancer 2015[epub ahead of print]. DOI:10.1007/ s00520-015-3053-6.

54. Cianfriglia F, Di Gregorio DA, Manieri A. Multiple primary tumours in patients with oral squamous cell carcinoma. Oral Oncol 1999;35:157-163.

55. Humphris GM, Rogers S, McNally D, LeeJones C, Brown J, Vaughan D. Fear of recurrence and possible cases of anxiety and depression in orofacial cancer patients. Int $J$ Oral Maxillofac Surg 2003;32:486-491.
56. Kissun D, Magennis P, Lowe D, Brown JS, Vaughan ED, Rogers SN. Timing and presentation of recurrent oral and oropharyngeal squamous cell carcinoma and awareness in the outpatient clinic. Br J Oral Maxillofac Surg 2006;44:371-376.

57. Thewes B, Butow P, Zachariae R, Christensen S, Simard S, Gotay C. Fear of cancer recurrence: a systematic literature review of self-report measures. Psychooncology 2012;21:571-587.

58. Llewellyn CD, Weinman J, McGurk M, Humphris G. Can we predict which head and neck cancer survivors develop fears of recurrence? J Psychosom Res 2008;65:525-532.

59. Mishel MH, Germino BB, Gil KM, et al. Benefits from an uncertainty management intervention for African-American and Caucasian older long-term breast cancer survivors. Psychooncology 2005;14:962-978.

60. Blank TO, Bellizzi KM. After prostate cancer: predictors of well-being among long-term prostate cancer survivors. Cancer 2006;106:2128-2135.

61. Kershaw TS, Mood DW, Newth G, et al. Longitudinal analysis of a model to predict quality of life in prostate cancer patients and their spouses. Ann Behav Med 2008;36:117-128.

62. Ackerstaff AH, Rasch CR, Balm AJM, et al. Five-year quality of life results of the randomized clinical phase III (RADPLAT) trial, comparing concomitant intra-arterial versus intravenous chemoradiotherapy in locally advanced head and neck cancer. Head Neck 2012;34:974-980.

63. Chen AM, Daly ME, Farwell DG, et al. Quality of life among long-term survivors of head and neck cancer treated by intensitymodulated radiotherapy. JAMA Otolaryngol Head Neck Surg 2014;140:129-133.

64. Payakatchett N, Ounpraseuth S, Suen JY. Late complications and long-term quality of life for survivors ( $>5$ years) with history of head and neck cancer. Head Neck 2013;35:819-825.

65. Taibi R, Lleshi A, Barzan L, et al. Head and neck cancer survivors patients and late effects related to oncologic treatment: update of literature. Eur Rev Med Pharmacol Sci 2014;18:1473-1481.

66. Powers AD, Oltmanns TF. Personality pathology as a risk factor for negative health perception. J Pers Disord 2013;27:359-370.

67. Williams PG, O'Brien CD, Colder CR. The effects of neuroticism and extraversion on self-assessed health and health-relevant cognition. Personal Individ Differ 2004;37:83-94.

68. Shah SB, Barsky AJ, Vaillant G, Waldinger RJ. Childhood environment as a predictor of perceived health status in late life. Health Psychol Res 2014;2:96-100.

\section{Supporting information}

Additional supporting information may be found in the online version of this article at the publisher's web site. 